Bull. Mater. Sci., Vol. 6, No. 2, May 1984, pp. 405-413. (C) Printed in India.

\title{
Some recent advances in materials technology
}

\author{
S RAMASESHAN and N BALASUBRAMANIAN* \\ Indian Institute of Science, Bangalore 560012, India. \\ *Everest Building Products, R\&D centre, Peenya II, Bangalore 560058, India.
}

\begin{abstract}
Recent advances in five key areas of materials technology are discussed. Structural and non-structural composites, electrically-conducting polymers, materials obtained by rapid quenching, new developments in hydraulic cements and photothermal solar energy conversion are reviewed.
\end{abstract}

Keywords. Composites; conducting polymers; metglasses; macroscopically defect free cement; selective surfaces.

\section{Introduction}

Recent years have seen vast strides in materials technology. The application of fundamental principles has improved materials by orders of magnitude and enabled them to meet contradictory requirements. For instance, permanent magnets with maximum energy product $(B H)_{\max }$ up to $240 \mathrm{~kJ}$ per cubic meter are commonplace today but in 1950 , only one fourth of this value had been attained. To cite another example one can now talk of making springs with cement. The methods of fabrication have also become sophisticated. In an effort to obtain more transistors per chip and larger computer memories at lower costs, the silicon technology has been improved to such an extent that it can now meet the threat from alternative technologies such as Josephson devices and magnetic bubbles. In the present review five growth areas are commented upon; the choice of topics to a certain extent depended upon the research interests of the authors.

\section{Composite materials}

\subsection{Structural applications}

The composite materials present the designer unique opportunities because of the possibility of (i) combining two or more phases with interesting properties and (ii) lamination of several plies to generate a laminate of optimum properties. The first topic is known as micromechanics (in the context of mechanical properties) and the second, as the laminated plate theory. Starting from the properties of the unidirectional composite where all the fibres are oriented along the direction of stress, the properties of off-axis composites and of laminates consisting of plies at different orientations can be calculated. In the case of elastic constants $Q_{i j}$, the calculations make use of the transformation relations for the fourth order tensors (Tsai 1983). The elastic constants of the laminate are obtained by applying the rule of mixtures relation to the elastic constants of the plies. For symmetric laminates which are thin, a $3 \times 3$ symmetric matrix 
of elastic constants is required to describe the elastic behaviour but for specially orthotropic laminates the number of constants required reduces to 4 .

The strength properties of anisotropic materials such as composites are described by a surface in strain ( $\varepsilon$ ) space (Tsai and Hahn 1980).

$$
G_{i j} \varepsilon_{i} \varepsilon_{j}+G_{i} \varepsilon_{i}=1 \text {. }
$$

The coefficients of (1) can be calculated from simple tests such as longitudinal tension and compression, transverse tension and compression and shear and the elastic constants discussed previously. The failure surfaces for plies with various orientations using (1) are shown in figure 1 for a graphite-epoxy composite. The inner envelope represents the minimum strength properties of this material. The use of such failure surfaces in the optimal design of composite laminates has been discussed elsewhere (Balasubramanian 1983). For a biaxial loading condition where the longitudinal tensile stress is twice the transverse one i.e., $\langle 2,1,0\rangle$, the optimum laminate will have 11 plies at $0^{\circ}$ and 5 plies at $90^{\circ}$ and will withstand $\langle 462,231,0\rangle \mathrm{MPa}$ before the first crack occurs. If glass-epoxy were used, 14 plies of $0^{\circ}$ and 4 plies of $90^{\circ}$ will be required. Such a laminate will withstand $\langle 108,54,0\rangle \mathrm{MPa}$ (Balasubramanian 1984). The decision to use one or the other can be made after taking into account costs, densities, thicknesses etc.

Such an ability to tailor the material along with the inherently high strength and stiffness of fibres and the easy processability of resins have led to the widespread use of composites. The Lear Fan 2100 uses carbon and Kevlar based composites which account for $70 \%$ of its weight (Noyes 1983). In the wing, cover skins, spars and ribs are made of carbon fibre woven fabrics. In the fuselage, main skin panels, pressure bulkheads, flooring and frames are made of fabrics and unidirectional tapes. The empennage contains horizontal and vertical stabiliser skins, main spars and ribs of carbon-composites. Aileron skins and spars, elevator skins and spars and flap skins contain Kevlar fabrics. For the same cost, the number of miles travelled on Lear fan 2100 is nearly 3.5 times that on Cessna citation. A number of other aerospace and engineering applications especially in India were reviewed earlier (Ramaseshan and Balasubramanian 1977).

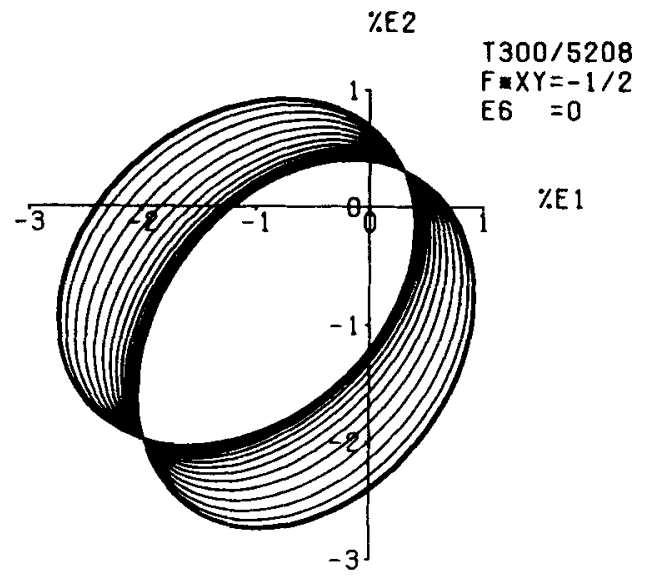

Figure 1. Failure surfaces of graphite-epoxy composite. 


\subsection{Non-structural applications}

Composite materials have interesting electrical, magnetic optical and thermal properties (Albers 1976). One class of materials is similar to the structural materials discussed above in which the constituents contribute proportionally. The second class consists of materials in which the physical output of one phase becomes the input for the second phase. Such properties have been called product properties (van Suchtelen 1972) or operator properties (Ramaseshan and Balasubramanian 1977). One example is a composite consisting of a piezomagnetic phase $A$ and a piezoelectric phase $B$. In such a composite, the application of a magnetic pulse induces a change in the shape of $A$. Since $\mathbf{A}$ and $\mathbf{B}$ are bonded together, $\mathrm{B}$ is also strained and being piezoelectric consequently develops an electric pulse. Thus, a magnetic pulse is converted into an electric pulse by the composite. The composite is the unidirectionally-solidified eutectic of barium titanate $\left(\mathrm{BaTiO}_{3}\right)$ which is the piezoelectric phase and the mixed nickel-iron oxide $\left(\mathrm{NiO}-\mathrm{Fe}_{2} \mathrm{O}_{3}\right)$ which is the piezomagnetic phase. Composites with operator properties can have entirely new properties or can give a known conversion but with a higher yield.

For optical applications, sodium fluoride-sodium chloride ( $\mathrm{NaF}-\mathrm{NaCl})$ eutectics can be solidified unidirectionally so that $\mathrm{NaF}$ rods are parallel to the axis of the specimen grown. The composite then has image transmission properties similar to those of fibreoptic materials. The spacing between the rods can be controlled by the conditions of solidification. In fact, the solidification in this system was studied under zero-gravity conditions in a space-processing experiment and the inter-rod spacing was found to be more uniform compared to the eutectic composites solidified on earth. The controlled eutectic can then act as a far infrared transmitting medium for wavelengths longer than the inter-rod distance (typically $6 \mu \mathrm{m}$ ). Other examples of non-structural composites include aligned indium antimonide-nickel antimonide eutectics which are currently in use as magneto-resistive and infrared materials (Weiss and Wilhelm 1963).

In structural applications one phase is used in fibre form (one-dimensional) and the other in bulk form (three-dimensional). In non-structural applications, interesting variations in properties are obtained by using the phases in various forms (powders, layers etc). The ceramic fabrication processes permit such variations (Newnham et al 1980). These authors use a coral skeleton characterised by (i) a narrow pore size distribution (ii) a pore volume which equals the volume of the solid phase and (iii) complete interconnectivity of pores.

The skeleton is machined to the desired geometry and vacuum-impregnated by wax. The skeleton is then leached out leaving the wax negative. The PZT slip (containing PZT, $2 \mu \mathrm{m}$ size, water and polyvinyl alcohol) is forced into the wax negative by vacuum impregnation. When the wax is burnt off, PzT in coral structure is obtained (this is the lost wax process, well-known in metallurgy). PZT is then filled with high purity silicone rubber and a composite of PZT/silicone is obtained. The composite has a longitudinal piezoelectric coefficient $\bar{d}_{33}$ of $160 \mathrm{pCN}^{-1}$. If now a biaxial compression is applied such that the sample height is reduced by $80 \%$ in one direction and by $20 \%$ in the perpendicular direction, the electric flux path through the piezoelectric is greatly reduced and the permittivity, $\bar{\varepsilon}_{33}$ is reduced. However $\bar{d}_{33}$ is reduced much less and the ratio between the two, the longitudinal piezoelectric voltage coefficient $\bar{g}_{33}=\left(\bar{d}_{33} / \bar{\varepsilon}_{33}\right)$ becomes very high, $300 \times 10^{-3} \mathrm{Vm} \mathrm{N}^{-1}$. This is 15 times the value for the homogeneous PZT transducer. 
The other examples of non-structural applications are wear-resistant composite coatings obtained by electroless and electrodeposition (Indira Rajagopal 1983) and ablative composites of high silica and phenolic (Ramachandran et al 1980).

\section{Conducting polymers}

Polymers are known for their processability, fracture toughness and low cost. They could provide cheaper alternative to metallic conductors if the polymers could be made conducting by the use of dopants. A list of conducting polymers produced this way is given in table 1 along with the data on their electrical conductivity. These materials are of considerable theoretical and practical interest (Heegar 1981; Mort 1980). Figure 2 shows the structure of polyacetylene, a non-pendant-group polymer with an unsaturated chain. Undoped polyacetylene (PA) is a semiconductor with a conductivity of $10^{-5}$ to $10^{-6} \mathrm{ohm}^{-1} \mathrm{~cm}^{-1}$. The conductivity is of the $p$ type as shown by thermopower measurements and the activation energy of the conductivity is $0.3 \mathrm{eV}$. Its band gap as deduced from optical absorption is $1.4 \mathrm{eV}$. When PA is doped by reacting the polymer with a strong oxidising or reducing agent, an excess of positive or negative charge is

Table 1. Conducting polymers

\begin{tabular}{|c|c|c|}
\hline Polymer & Dopant & $\begin{array}{l}\text { Conductivity } \\
\left(\mathrm{ohm}^{-1} \mathrm{~cm}^{-1}\right)\end{array}$ \\
\hline Polyacetylene & Arsenic pentafluoride & 1200 \\
\hline Poly-p-phenylene & Arsenic pentafluoride & 500 \\
\hline Polypyrrole & Iodine & 100 \\
\hline Polypyrrole & Perchlorate & 40 \\
\hline $\begin{array}{l}\text { Polyaluminophthal- } \\
\text { ocyanine fluoride }\end{array}$ & Iodine & $4 \cdot 5$ \\
\hline $\begin{array}{l}\text { Polyphthalocya- } \\
\text { nineallorane }\end{array}$ & Iodine & $1 \cdot 4$ \\
\hline Polyphenylene sulfide & Arsenic pentafluoride & 1 \\
\hline Mercury & - & $10^{4}$ \\
\hline Copper & - & $6 \times 10^{5}$ \\
\hline
\end{tabular}<smiles>C/C=C\C=C/C=C\C</smiles>

Figure 2. Structure of polyacetylene.<smiles>C/C=C\C=C/C=C\C(=O)[O-]</smiles> 
produced on the polymer. The charge is free to move through the polymers because of the inter linking of double bonds. Specifically, PA doped with $\mathrm{AsF}_{5}$ or with iodine (electron acceptors) has a conductivity of $1000 \mathrm{ohm}^{-1} \mathrm{~cm}^{-1}$. Doping with sodium napthalide (electron donor) leads to $n$-type material with similar conductivity. The behaviour is concentration-dependent. At low doping levels, the material is a semiconductor and at 1 to 3 atomic $\%$ of doping, transition to metallic behaviour takes place. There are two proposed models. According to the first, the transition is due to percolation of $(\mathrm{CH})_{x}$ chains. These constitute the conducting particles in an insulator and after sufficient doping, interconnections between conducting particles occur (Zallen 1978). In the second model soliton (solitary wave solutions to nonlinear problems) theory of electrical conduction is used. At low doping levels, it is energetically more favourable for the charge from the dopant molecule to bind itself to the soliton rather than enter the conduction or valence bands ( $\mathrm{Su}$ et al 1979). At higher doping levels solitons become so numerous that they overlap to form a continuous metallic state. ESR spectra of trans-PA confirm the presence of one neutral soliton for every 3000 bonds.

The practical applications of conducting polymers include (1) photoreceptor elements (2) photovoltaic cells and (3) commercial batteries. However, there is need for considerable development work. New ways of doping have to be found to assure the stability of conductors in air. The structural irregularities in the resin affect conductivity and will have to be minimised. There is also evidence that the solutions of lithium iodide in dimethoxyethane (DME), intended for use in light weight batteries leach out the triiodide ions $I_{3}$ (produced by the action of $I_{2}$ on PA) from PA. When this happens the conductivity of the iodine-doped PA drops by eight orders of magnitude. The same phenomenon occurs while charging the battery. Hence a barrier coating which prevents leaching without affecting the conductivity will have to be developed.

\section{Materials by rapid solidification}

Duwez and coworkers (Klements et al 1960) showed that some alloys could be cooled fast enough $\left(10^{6}{ }^{\circ} \mathrm{C} \mathrm{sec}-1\right)$ to yield glasses (Metglasses). These are now produced commercially. The liquid metal can be passed through a nozzle with a thin slit that lies in close proximity to a cold rotating wheel. As the metal flows from the slit it is very quickly quenched into a glass (Narasimhan 1979). This process can, in principle, produce any desired width. Metglasses mostly contain an early or late transition metal and a metalloid (Suryanarayana et al 1980) or an early transition metal and a late transition metal (Suryanarayana 1980). Fast cooling can produce new crystalline phases or amorphous alloys. The high pressure annealing of amorphous alloys can yield new crystalline phases which are not obtained by annealing at ambient pressures. Rapid solidification processing eliminates forging, rolling, drawing etc., and is much faster compared to the conventional processes. However, the heat extraction must be rapid which limits the shape of articles produced to ribbons, wires and thin sheets. They also are unstable at high temperatures. The corrosion rate of Metglasses is 1000 times smaller than that of stainless steels. Ferrous glasses are easily magnetized and exhibit high saturation magnetizations. The loss factor is very low (Luborsky et al 1978). Although a number of structural applications (Gilman 1980) have been suggested including reinforcements in composites (Strife and Prewo 1981; Yeow 1980) Metglasses 
are important from the point of view of magnetic properties. The use of metallic glass cores in power transformers will reduce core losses and result in energy savings. The use of lasers to melt only the surface of the material or laser glazing is an interesting development. A laser melts the surface of a bulk material which rapidly solidifies when the incident beam is removed. The otherwise conventional material now has a surface layer 1 to $10 \mathrm{~mm}$ thick which is amorphous or microcrystalline in nature.

\section{Macroscopically defect-free (MDF) cement}

Cement and steel are the most widely used construction materials. But our understanding of cement is much poorer than that of steel. This situation is changing as a result of interdisciplinary research devoted to cement. The hydraulic cements (ordinary portland cement and its modifications, calcium aluminate cement etc.) are inert, noncombustible and cost less energy to produce them than metals or plastics. It is easy to shape them into finished parts because the setting takes place at room temperature. However the tensile strength and strain to failure and fracture toughness are low compared to other materials. This is why cement is used in compression. The limitations of cement have been overcome by the modification of their structure (Birchall et al 1981, 1982). The conventional cement has a porosity of 25 to $35 \%$ and pore sizes of $10^{-9}$ to $10^{-3} \mathrm{~m}$. It was previously thought that the low strength of cement could be explained entirely by the porosity dependence of strength (Feret 1897). It is now realised that the pore acts like a Griffith flaw and the pore size affects the strength more strongly (Kendall et al 1983). Simple equations to quantify these ideas have been available in the literature for a long time. The strength $(\sigma)$ dependence on flaw size $(c)$ is given by the Griffith (1920) formula

$$
\sigma=(E R / \pi c)^{1 / 2},
$$

where $E$ is the Young's modulus and $R$ the fracture energy. For a solid of porosity $p$

and

$$
E=E_{0}(1-p)^{3} \text {, }
$$

where $k$ is a constant. Hence

$$
\sigma=\left(\frac{E_{0} R_{0}}{\pi c}\right)^{\frac{1}{2}}\left[(1-p)^{3} \exp (-k p)\right]^{1 / 2}
$$

Equation (5) suggests that the strength can be improved by (i) reducing porosity $p$ (ii) reducing the pore size, $c$ and most importantly, (5) shows that the reduction of porosity by $30 \%$ can increase the strength by a factor of 2 , but the reduction of pore size from $1 \mathrm{~mm}$ to $0.01 \mathrm{~mm}(10 \mu \mathrm{m})$ increases the strength by a factor of 10 .

By the addition of a polymer, dough mixing and air removal it is possible to produce cement with pore size less than $15 \mu \mathrm{m}$. Such a cement is known as the Macroscopically Defect Free cement (Birchall et al 1981). The properties of such cement are shown in table 2 along with those of ordinary cement for comparison. The data show that the MDF is not only vastly superior to the conventional cement but can compete with aluminium and steel in many applications.

The following water-soluble polymers have been used in making the MDF cement: 
Table 2. Properties of MDF compared to ordinary cement

\begin{tabular}{|c|c|c|}
\hline & $\begin{array}{l}\text { Ordinary } \\
\text { cement }\end{array}$ & $\begin{array}{c}\text { MDF } \\
\text { cement }\end{array}$ \\
\hline Flexural strength, $\mathrm{MPa}$ & 10 & $40-150$ \\
\hline Young Modulus, GPa & 20 & $35-50$ \\
\hline Compressive strength, $\mathrm{MPa}$ & 40 & $100-300$ \\
\hline Fracture energy $\left(\mathrm{J} \mathrm{m}^{-2}\right)$ & 20 & $40-200$ \\
\hline Electrical breakdown $\left(\mathrm{k} V \mathrm{~mm}^{-1}\right)$ & 0.1 & $6-13$ \\
\hline Gas permeability (mol m-1 $\sec ^{-1} \mathrm{~Pa}^{-1}$ ) & $10^{-12}$ & $10^{-16}$ \\
\hline
\end{tabular}

(i) hydroxy propylmethyl cellulose (ii) polyacrylamide (iii) polyvinyl alcohol and (iv) polyvinyl acetate.

A typical composition is given by 100 parts (by mass) cement, 7 parts polymer and 10 parts water. This materials is thoroughly mixed by applying shear. The polymer attaches to the cement particles and acts as a lubricant promoting the sliding of the cement particles so that they can be closely packed. By applying a low pressure (5 MPa) air bubbles can be removed. The space available for the growth of hydration products is restricted due to close packing in MDF cement paste and calcium hydroxide is present as a platlet, $10 \mathrm{~mm}$ thick and larger crystals may act as flaws and reduce the strength. The MDF cement will find many applications in the building industry in the form of sheets, pipes, rods, $T$-sections etc. With fibre reinforcement (e.g. Kevlar) further improvements in fracture toughness are possible.

\section{Surfaces for selective absorption of solar energy}

Efforts are being made to convert solar energy into useful heat. The simplest device which does that is the flat plate collector, which has a black surface painted on it. But the radiation losses from such a surface are considerable. Hence a spectrally selective surface which absorbs solar energy without radiating heat is efficient. For efficient photothermal conversion of solar energy, the absorbing surface should ideally exhibit zero reflectance over the solar range $(0 \cdot 3 \leqslant \lambda \leqslant 2 \mu \mathrm{m})$ and unit reflectance over the thermal range ( $2 \lesssim \lambda \lesssim 20 \mu \mathrm{m}$ ) (figure 3 ). The reflectance should change at a wavelength $\lambda_{c}$ of say $3 \mu \mathrm{m}$. An example of a material with such selective absorption is $\mathrm{Zr} \mathrm{B}_{2}$.

More frequently a composite of a semiconductor and metal is used. The short

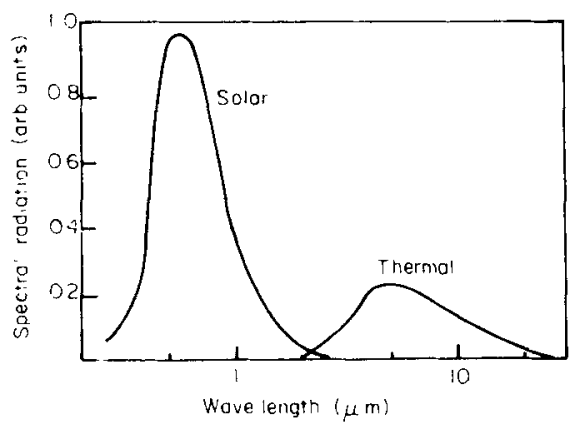

Figure 3. Solar and thermal spectra. 


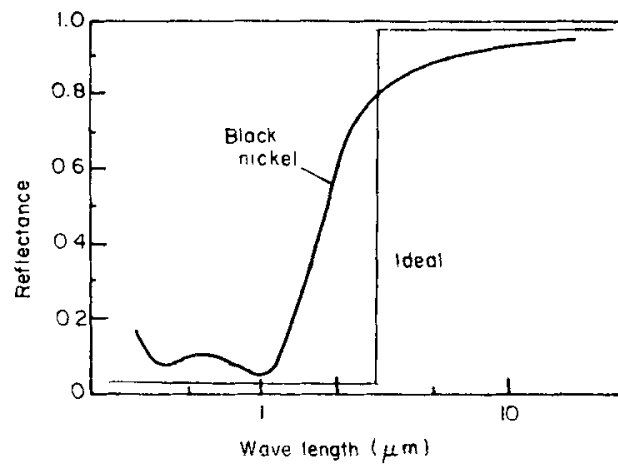

Figure 4. Spectral reflectance of the ideal and an actual selective surface.

wavelength radiation can be absorbed by the semiconductor of appropriate bandgap $(\approx 0.5 \mathrm{eV})$ and the metal substrate has a low thermal emittance. Figure 4 shows the behaviour of such a composite along with that of the ideal selective surface.

The most widely used surfaces are: (i) "black chrome". This is a $\mathrm{Cr}-\mathrm{Cr}_{2} \mathrm{O}_{3}$ composite produced by electroplating. (ii) Nickel, pigmented $\mathrm{Al}_{2} \mathrm{O}_{3}$. This is a $\mathrm{Ni}-\mathrm{Al}_{2} \mathrm{O}_{3}$ composite produced by the electrolytic colouration of anodized aluminium sheet. This surface has a solar absorptance of 92 to $97 \%$ and thermal emittance of 10 to $26 \%$. (iii) $\mathrm{Ni}-\mathrm{NiO}$ composite produced by chemical conversion of nickel foil. A coevaporated $\mathrm{Mo}-\mathrm{Al}_{2} \mathrm{O}_{3}$ composite layer coated with $\mathrm{Al}_{2} \mathrm{O}_{3}$ and deposited on to molybdenum has a solar absorptance of $99.2 \%$ and normal thermal emittance below $8 \%$. The promising coatings for photo thermal energy conversion must be assessed for long term durability in operating environments (Bogaerts and Lampert 1983).

\section{Concluding remarks}

While commenting on the frontier technologies, one must be aware that threats could come out of the blue and cause panic. This happened in the case of graphite-epoxy composites when the moisture problem arose and again when the graphite fibres released from the composite caused electrical short-circuits. In both cases the probability of failure was shown to be low. In the case of fibre-optic cables, hydrogen was found to diffuse through the nylon and steel cladding and concentrate inside the cable. It could diffuse into the quartz fibre and attenuate light especially at long wavelengths. This danger is present in submarine cables where the protective steel cladding could set up a galvanic cell with the sea water and produce hydrogen. It was discovered that the caulking compound used to seal the under water joints also had the property of blocking the hydrogen gas. The areas we have chosen to discuss are the ones where science and technology have progressed together and where it is difficult to halt the progress merely because of the fear of the unknown.

\section{References}

Albers W 1976 Composite materials, Proc. Int. Conf. (ed.) E Scale (New York: AIME) Vol 2 Balasubramanian N 1983 Curr. Sci. 521171

Balasubramanian N 1984 to be published 
Birchall J D, Howard A J and Kendall K 1981 European Patent No. 021682

Birchall J D, Howard A J and Kendall K 1982 European Patent No. 0055035

Birchall J D 1983 Philos. Trans. R. Soc. London A310 p 31

Bogaet ts W F and Lampert C M 1983 J. Mater. Sci. 102847

Feret R 1897 Bull Soc. Encour. Ind. Natl Paris II 1604

Gilman J J 1980 Science 80856

Griffith A A 1920 Philos. Trans. R. Soc. London A221 163

Heeger A J 1981 Commun. Solid State Phys. 1053

Indira Rajagopal 1983 Bull. Mater. Sci. 5323

Kendall K, Howard A J and Birchall J D 1983 Philos. Trans. R. Soc. London A310 139

Klements W, Willens R H and Duwez P 1960 Nature (London) 187869

Luborsky F E, Frischmann P G and Johnson L A 1978 J. Magn. Mater. 8318

Mort J 1980 Science 80 p 819

Narasimhan D 1979 U S Patent 4142571

Newnham R E, Bowen L J, Klicker K A and Cross L E 1980 Mater. Eng. 293

Noyes J V 1983 Composites 14129

Ramachandran B E, Pai B C and Balasubramanian N 1980 J. Am. Cer. Soc. 631

Ramaseshan S and Baiasubramanian N 1977 Sci. Today Sept. Issue

Strife and Prewo K M 1981 Comp. Tech. Rev. 376

Su W P, Schrieffer J R and Heeger A J 1979 Phys. Rev. Lett. 421698

Suryanarayana C 1980 Rapidly quenched metals-A bibliography 1973-79 (New York: IFI/Plenum)

Suryanarayana C, Inoue A and Masumato T 1980 J. Mater. Sci. 151993

Tsai S W 1983 ICM-4 (eds) J Carlsson and N G Ohlson (New York: Pergamon) Vol 4 p 103

Tsai S W and Hahn H T 1980 Introduction to composite materials (Pennsylvania: Technomic)

Van Suchtelen J 1972 Phillips Res. Rep. 2728

Weiss H and Wilhelm M 1963 Z. Phys. 176399

Yeow Y T 1980 Comp. Tech. Rev. 217

Zallen 1978 Proc. 13th IUPAP Conf. on Statistical Physics (eds) C G Kuper and I Riess (Bristol: Hilger) 